\title{
Evaluation of Texture Maps as Input to Extract Deep Features in Glaucoma Diagnosis
}

\author{
Daniel Veloso da Silva ${ }^{1}$, Romuere Rodrigues Veloso e Silva $a^{1,3,4}$ \\ ${ }^{1}$ Sistemas de Informação, Campus Senador Helvídio Nunes de Barros \\ Universidade Federal do Piauí (UFPI), Picos - PI - Brasil \\ ${ }^{2}$ Programa de Pós-Graduação em Engenharia Elétrica \\ Universidade Federal do Piauí (UFPI), Teresina - PI - Brasil \\ ${ }^{3}$ Programa de Pós-Graduação em Ciência da Computação \\ Universidade Federal do Piauí (UFPI), Teresina - PI - Brasil \\ \{danielveloso, romuere\}@ufpi.edu.br
}

\begin{abstract}
Glaucoma is a significant cause of blindness in the world. Doctors use computerized images to detect these diseases. Early detection of the disease increases the chances of treatment, reducing the adverse effects. This work proposes an evaluation of texture maps combinations as input to Convolutional Neural Networks for glaucoma classification in retinal images. In our experiments, we used three textures maps, three CNN architectures, and three classifiers. We achieve a Kappa $=0.708 \pm 0.054$ and a Accuracy $=0.859 \pm 0.021$. We conclude that using the combination of texture maps can improve the automatic detection of glaucoma compared to single-channel inputs, and could be used by state-of-the-art methods to improve their classification rates.
\end{abstract}

\section{Introdução}

A tecnologia vem sendo aplicada na resolução de problemas em diversas áreas do conhecimento. Sistemas inteligentes veem sendo empregados para auxiliar profissionais em diversos cenários. Neste trabalho abordaremos a utilização da tecnologia na área da saúde, frisando a detecção precoce do glaucoma.

O glaucoma é uma doença progressiva, e o não tratamento da mesma pode resultar na perda da visão. Dados divulgados pela Organização Mundial de Saúde(OMS) apresentam esta doença como uma das principais causas de cegueira mundial. Uma pesquisa realizada pela OMS revela que $15 \%$ da cegueira existente no mundo é causada pelo glaucoma [Thylefors and Negrel 1994]. O glaucoma pode se apresentar de 4 tipos diferentes, sendo eles glaucoma de ângulo fechado (agudo), glaucoma de ângulo aberto (crônico), glaucoma congênito e glaucoma secundário. Embora o glaucoma não possua uma cura, todos os tipos apresentados possuem tratamento eficaz quando detectado precocemente.

O glaucoma é causado por uma lesão no Disco Óptico (DO), que é responsável por transmitir o que vemos ao cérebro [LENSCOPE 2018]. Usualmente o glaucoma é diagnosticado em uma imagem e para isso é necessário delimitar a região do DO e da cavidade do DO (CDO), que é a região mais interna. O indicador mais utilizado para determinar a presença do glaucoma é o aumento do CDO. Para isso, calculada-se o Cupto-Disc Ratio (CDR), que é a razão entre o $\mathrm{CDO}$ e o $\mathrm{DO}$, quanto mais próximo de 1 maior a probabilidade da presença do glaucoma. 


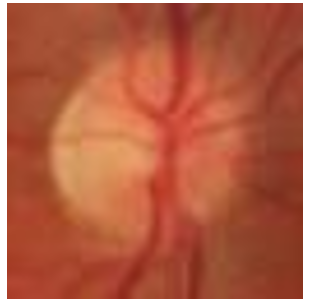

(a)

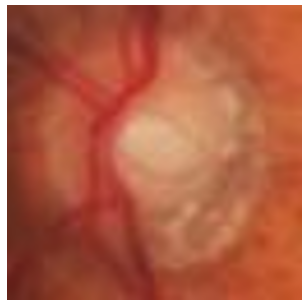

(b)

Figura 1. Exemplo de imagens do disco óptico saudável (a) e glaucomatosa (b).

Apesar do CRD ser uma forma bastante utilizada e confiável para detectar automaticamente o glaucoma em imagens, ele requer que as delimitações do DO e do CDO sejam feitas a priori. Estas necessitam de um grau de precisão próximo ao real para que o cálculo do CRD seja confiável. Dessa forma, a fim de ajudar na identificação da doença em seus estágios iniciais, desenvolvemos uma proposta de identificação do glaucoma, utilizando descritores de textura combinados a Redes Neurais Convolucionais (Convolutional Neural Network - CNN que não necessitam da etapa de segmentação de estruturas previamente.

Está proposta desenvolvida não possuí o objetivo de ultrapassar o estado da arte, visamos avaliar se outro tipo de entrada em redes neurais melhoram os resultados quando comparados com a imagem original. Os resultados obtidos com o método desenvolvido, visão ajudar os especialistas no diagnóstico, oferecendo uma segunda opinião.

\section{Trabalhos Relacionados}

Os trabalhos relacionados expostos nesta seção, apresentam as diversas técnicas utilizadas no campo de visão computacional com enfase na detecção de glaucoma.

O trabalho apresentado por [Li et al. 2018], utiliza o conjunto de dados LabelMe2016, de onde foram retiradas 70.000 imagens das 200.000 existentes para uma amostragem aleatória, e selecionaram 48.116 imagens para a rotulagem de um algoritmo de aprendizado para a detecção da neuropatia óptica glaucomatosa. Os autores realizaram a normalização das imagens com os pixeis no intervalo de 0 a 1 , e a redução para uma matriz de 299x299. Com isso obtiveram área sob a curva ROC (AUC) de 0,986, sensibilidade (Sens) de 95,6\% e especificidade (Espec) de 92\%.

O trabalho [Raghavendra et al. 2018] utilizou uma CNN de 18 camadas, proposta pelos mesmos. Dividiram o banco de dados em treino e teste. O banco de dados utilizado é o Kasturba Medical College, Manipal India, com um total de 589 imagens normais e 837 com glaucoma, onde com as mesmas obtiveram uma acurácia (Acc) de 98,13\%. Para o desenvolvimento deste trabalho, os autores criaram a conjunto de dados, os quais não estão disponíveis.

O trabalho apresentado em [Araujo 2018], aborda uma metodologia para diagnóstico do glaucoma a partir das imagens de fundo de olho, utilizando os índices de diversidade, como descritores de textura para a extração das características de textura. Os algoritmos genéticos são utilizados para seleção das características mais relevantes. Após a extração das características, os autores utilizaram a máquina de vetores de suporte (SVM) para realizar a classificação obtendo acurácia de 93,41\%, sensibilidade de 92,36\% 
e especificidade de $95,05 \%$.

O trabalho de Júnior et al em [Carvalho-Júnior et al. 2017], desenvolveu um método para detecção automática do glaucoma com imagens da retina utilizando imagens do banco de dados RIM-ONE. Utilizaram a técnica de segmentação do Disco óptico aplicando o algoritmo de Otsu. Para a extração de características utilizaram descritores de textura baseados em índices de diversidade filogenética para classificar utilizando o framework WEKA. Os resultados apresentados foram acurácia de 98,6\%, sensibilidade 92,9\% e Especificidade de $100 \%$.

No artigo [SILVA et al. 2018] é proposto uma metodologia para o diagnóstico do glaucoma através da análise da textura da imagem representada usando LBP (Local Binary Pattern) e estatística espacial aplicada em imagens médicas de retinografias. Os autores obtiveram acurácia de $95.08 \%$. O método foi aplicado em imagens segmentadas baseada em especialista que continha na base de imagem RIM-ONE.

No trabalho [Al-Bander et al. 2017] foi empregada uma metodologia para a detecção do glaucoma utilizando redes neurais e o classificador SVM. Em seu trabalho é utilizado o banco de dados RIM-ONE, onde foi realizado o redimensionamento das imagens para 227x227 pixels. Utilizou uma CNN pré-treinada (Alexnet) para a extração de características e por fim e utilizou o classificador SVM, obtendo assim acurácia de $88,2 \%$, sensibilidade $85 \%$ e especificidade de $90,8 \%$.

O trabalho [Ahn et al. 2018] utiliza em seu desenvolvimento um banco de dados privado. A sua metodologia consiste na utilização da CNN GoogleNet Inception v3. como extrator e classificador de características. Neste trabalho os autores redimensionaram as imagens na largura fixa de 800 pixels, e logo em seguida utilizaram uma cnn's pré treinada para a extração e classificação de características. O Resultado apresentado pelos autores foi uma AUC de 0,93.

A Tabela 1 apresenta um resumo dos trabalhos relacionados, mostrando a base de dados utilizada e os resultados obtidos.

Tabela 1. Comparativo dos trabalhos do estado da arte.

\begin{tabular}{llcccc}
\hline Autor & $\begin{array}{l}\text { Base de } \\
\text { Imagens }\end{array}$ & Acc (\%) & AUC & Sens (\%) & Espec (\%) \\
\hline [Li et al. 2018] & $\begin{array}{l}\text { LabelMe } \\
\text { 2016 }\end{array}$ & - & 0,99 & 95,60 & 92,00 \\
\hline [Raghavendra et al. 2018] & $\begin{array}{l}\text { Kasturba } \\
\text { Medical } \\
\text { College }\end{array}$ & 98,13 & - & - & - \\
& RIM-ONE & 93,41 & - & 92,36 & 95,05 \\
\hline VAraujo 2018] & RIM-ONE & 98,60 & - & 92,90 & 100 \\
\hline [Carvalho-Júnior et al. 2017] & RIM-On & & \\
\hline [SILVA et al. 2018] & RIM-ONE & 95,08 & - & - & - \\
\hline [Al-Bander et al. 2017] & RIM-ONE & 88,20 & - & 85,00 & 90,80 \\
\hline [Ahn et al. 2018] & Privada & - & 0,93 & - & - \\
\hline
\end{tabular}


Utilizamos no presente trabalho, três algoritmos de textura e três tipos de CNNs diferentes. Realizamos os testes em um conjunto de dados público disponível para a replicação de testes. Classificamos as características com três algoritmos diferentes. Considerando o atual artigo com os demais podemos destacar uma maior exploração das técnicas de entrada de dados nas CNNs.

\section{Materiais e Métodos}

Neste trabalho foram utilizados conhecimentos das áreas de visão computacional com aplicação de mapas de textura na extração de informações obtidas através de imagens retinográficas. Nas seções seguintes, serão descritas a base de dados, os métodos para extração dos mapas de textura e de atributos, bem como a técnica utilizada para a classificação das imagens.

\subsection{Base de Imagens}

Neste trabalhos utilizamos a base de imagens RIM-ONE-V2, ela consiste em imagens fornecidas por três hospitais. Sua composição consiste em 455 imagens, sendo 200 glaucomatosas e 255 saudáveis [RIMONE 2015]. Na Figura 2, apresentamos duas amostras de ambas as imagens contidas no RIM-ONE.

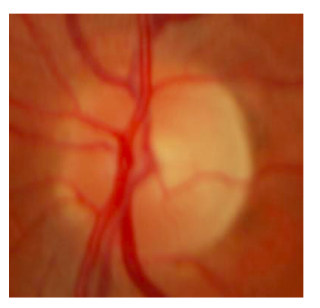

(a)

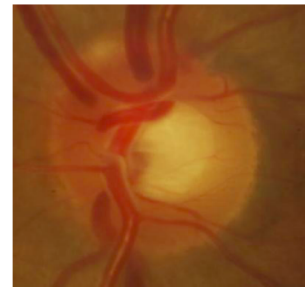

(b)

Figura 2. Exemplos de imagens da base de dados RIM-ONE-V2. Em (a) uma imagem de uma retina saudável e em (b) uma glaucomatosa.

\subsection{Mapas de Textura}

A análise de mapas de textura é um método quantitativo de pré-processamento para extração de dados [Stanzione et al. 2019]. Nesta seção serão apresentados algoritmos clássicos para obtenção de mapas de textura e que foram utilizados neste trabalho.

\subsection{Wavelet - WAV}

A transformada Wavelet é comumente usada em muitos problemas de visão computacional. No WAV é realizada uma decomposição de wavelet 2-D de nível único, que requer uma função de dimensionamento 2-D, e três wavelets $2-\mathrm{D}$, onde o índice $\mathrm{i}=\mathrm{H}, \mathrm{V}, \mathrm{D}$ é a representação das variações de intensidade ao longo de colunas ou arestas horizontais e ao longo das diagonais [Mallat 1989]. A Figura 3 mostra a aplicação do descritor em uma das imagens do banco de dados RIM-ONE-V2.

\subsection{Gray Level Co-occurrence Matriz - GLCM}

A Matriz de Co-ocorrência de Nível de Cinza (GLCM) é um histograma de valores de escala de cinza coocorrência em um determinado deslocamento sobre uma imagem. $\mathrm{O}$ 


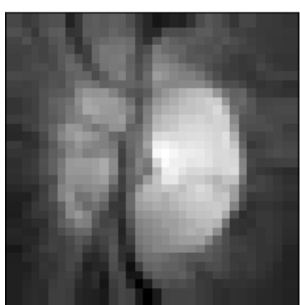

(a)

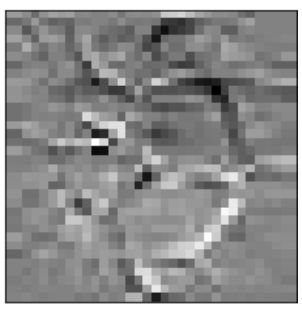

(b)

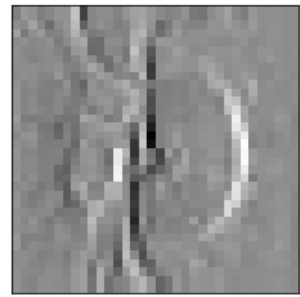

(c)

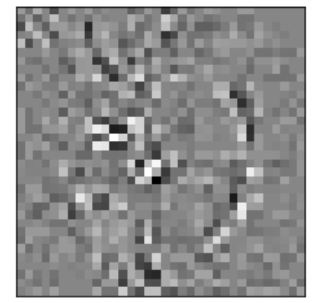

(d)

Figura 3. Imagem com células glaucomatosa da Base RIM-ONE com a aplicação do WAV. (a) Aproximação, (b) Detalhe horizontal, (c) Detalhe vertical, (d) Detalhe diagonal.

presente algoritmo mostra com que frequência cada nível de cinza ocorre em um pixel localizado em uma posição geométrica fixa relativa a cada outro pixel [Haralick et al. 1979]. Essa frequência gera uma matriz de coocorrência, que será utilizada como mapas de textura. A média da GLCM nas 4 direções $\left(0^{\circ}, 45^{\circ}, 90^{\circ}, 135^{\circ}\right)$, usando a distância $=1$.

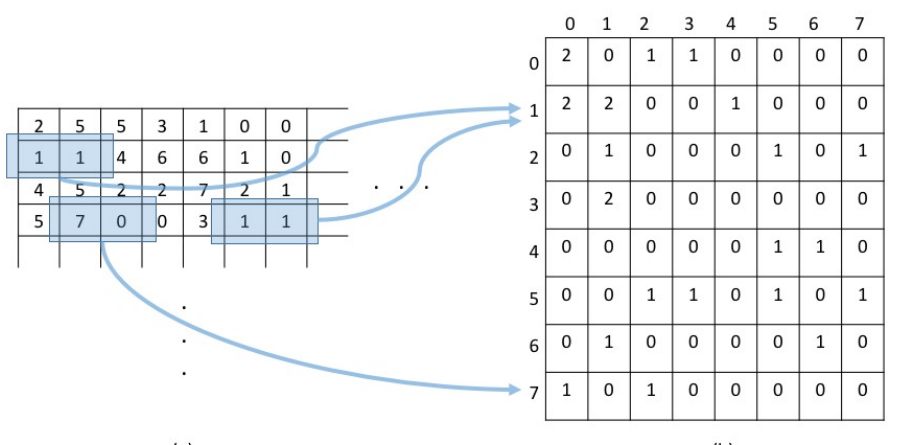

(b)

Figura 4. Funcionamento do descritor GLCM. (a) imagem, (b) matriz GLCM. Fonte: [SILVA et al. 2017]

\subsection{Histogram of Oriented Gradients - HOG}

O Histograma de Orientação dos Gradientes (HOG) combina a descrição de características de forma e textura. Ele preconiza que a aparência e forma de um objeto local pode ser distinguida pelas direções das bordas ou pela distribuição do gradiente da intensidade. As etapas do HOG consistem em uma normalização de imagem (opcional), computação dos gradientes para obtenção da magnitude e direção. Em seguida são computados os histogramas de gradiente a partir de células [Dalal and Triggs 2005].

Ao final é realizada uma normalização de blocos para obtenção do vetor de atributos. Os parâmetros de entrada do HOG são quantidade de células, blocos. Neste trabalho utilizamos 3 células e 3 blocos. A Figura 5 é uma imagem com a aplicação do descritor.

\subsection{Redes Neurais Convolucionais}

Uma rede neural convolucional ( $\mathrm{CNN}$ ou ConvNet) é um tipo especial de redes neurais multicamada, projetadas para reconhecer padrões visuais diretamente de imagens de pixel com pré-processamento mínimo. Neste trabalho utilizamos três arquiteturas de CNNs, as 


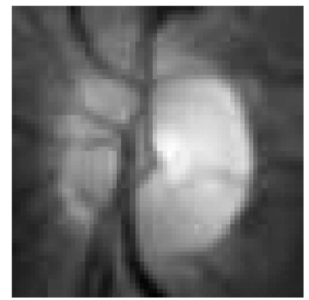

(a)

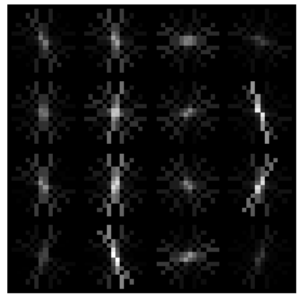

(b)

Figura 5. Imagem com células glaucomatosa da Base RIM-ONE com a aplicação do HOG. (a) Imagem em tons de cinza, (b) Mapa de textura HOG.

mesmas foram escolhidas pela frequência com que são utilizadas em trabalhos relacionados. A seguir mostramos algumas informações sobre cada uma.

As redes VGG16 e VGG19 [Simonyan and Zisserman 2014] são caracterizadas pela sua simplicidade, usando apenas camadas convolucionais $3 \times 3$ empilhadas umas sobre as outras em profundidade crescente. A redução do tamanho do volume é tratada pelo pool máximo. Duas camadas totalmente conectadas, cada uma com 4.096 nós. As redes VGG16 e VGG19 Os "16" e "19" representam o número de camadas de peso na rede. Todas as características extraídas para a classificação foram retiradas da penúltima camada da CNN.

A ResNet50 é uma CNN apresentada por [Huang et al. 2016] é baseada em redes simples, que insere "atalhos"fazendo com que a rede se torne residual, as saídas de uma camada são entradas de camadas mais a frente. Os atalhos podem ser aplicadas quando as entradas e as saídas tem as mesmas dimensões, mesmo não tendo as mesmas dimensões tem tratamentos.

\section{Metodologia}

O método proposto consiste em 5 etapas, apresentadas na Figura 6: a primeira etapa é a aquisição de imagens, onde utilizamos a base de dados RIM-ONE-V2; após isso, temos a etapa de geração dos mapas de textura onde utilizamos WAV, GLCM e HOG; o passo seguinte foi utilizar esse mapas de textura como entrada para as CNNs, foram utilizadas as redes VGG16, VGG19 e ResNet50; a última etapa foi a classificação das imagens onde utilizamos os atributos extraídos pelas CNNs e classificamos nosso conjuntos de dados em saudáveis e glaucomatosas.

\subsection{Cálculo dos Mapas}

As técnicas explicadas na Seção 3 foram aplicadas no banco de dados publico RIM-ONE V2. A primeira técnica aplicada nas imagens, foram os descritores de textura. Utilizamos os descritores como geradores de mapas de textura, assim aplicamos em todas as imagens 3 descritores que já foram mencionados na seção anterior, gerando assim 3 amostras de imagens, uma com um descritor.

Com os mapas de texturas, utilizamos a ideia da combinação de 2 mapas de textura com uma imagem em níveis de cinza para a criação de uma nova imagem. A ideia consiste na utilização da estrutura de uma imagem RGB, porém ao invés de utilizar a imagem colorida, optamos por colocar mapas de texturas por canais. De forma abstrata a Figura 7 representa a metodologia utilizada para geração da nova imagem com os mapas de textura. 


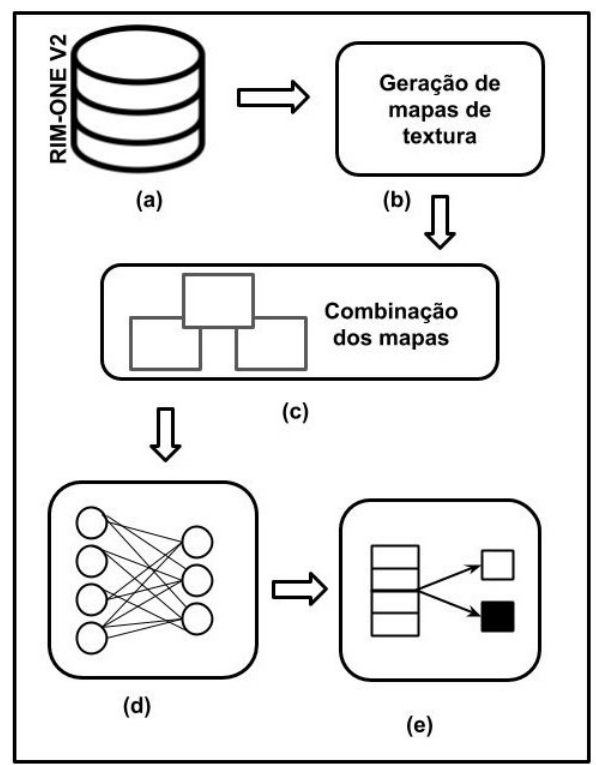

Figura 6. Metodologia proposta. (a) Banco de Dados RIM-ONE V2. (b) Aplicação dos mapas de textura. (c) Combinação dos mapas de textura. (d) Extração das características utilizando CNNs. (e) Classificação.

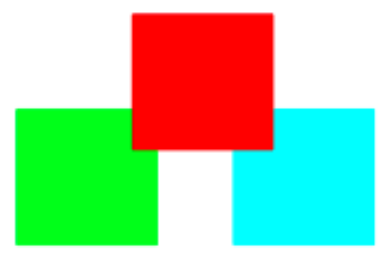

(a)

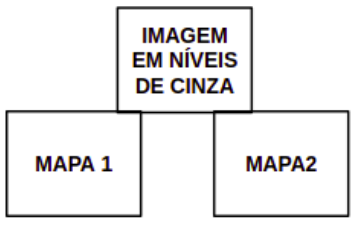

(b)

Figura 7. Reconstrução da Imagem. (a) llustração dos canais RGB (b) Reconstrução da imagem com os mapas de texturas.

Com os três descritores de texturas conseguimos obter três combinações: 1) WAV + imagem em níveis de cinza + HOG (WCH), 2) GLCM + imagem em níveis de cinza + HOG (GCH), e 3) WAV + imagem em níveis de cinza + GLCM (WCG).

A imagem em níveis de cinza utilizada para montar as combinações foi obtida pela Equação 1, que é o padrão mais utilizado em aplicações de processamento digital de imagens [Recommendation ].

$$
\text { Cinza }=0.2126 * R+0.7154 * G+0.0721 * B
$$

onde R, G e B são os canais vermelho, verde e azul do modelo de cores RGB.

\subsection{Extração de Características}

Logo em seguida, utilizamos as três CNNs para extrair características e por fim classificar as imagens. Utilizamos a metodologia de transferência de aprendizado para extrair atributos com as CNN. Para isso, utilizamos os pesos da base de imagens ImageNet [Deng et al. 2009]. Com isso, não foi necessário treinar as redes com os dados de imagens com e sem glaucoma. 
Da mesma forma que os descritores, utilizamos as CNNs uma por vez, gerando, assim, os descritores para serem classificados.

\subsection{Classificação}

Para o processo de classificação, utilizamos três algoritmos da literatura, são eles: Gaussian Processes (GP) [Rasmussen 2003], Decision Tree (DT) [Breiman et al. 1984], Random Forest (RF) [Breiman 2001].

No classificador Gaussian Processes utilizamos o otimizador L-BFGS-B e 100 como quantidade máxima de iteração para a predição. Para a Decision Tree utilizamos o índice Gini como função para medir a qualidade das divisões dos nós e a quantidade mínima de atributos para criar um novo nó foi 2 . No classificador Random Forest, utilizamos 100 árvores de decisão e o índice Gini como função para medir a qualidade das divisões dos nós.

$\mathrm{Na}$ etapa de treinamento dos classificadores utilizamos $80 \%$ dos dados para treinamento e $20 \%$ para teste. Além disso, executamos cada classificação 10 vezes sempre modificando os conjuntos de treinamento e teste. Com isso, obtivemos para cada métrica utilizada uma média e o desvio padrão das 10 execuções.

Utilizamos como métricas de avaliação dos métodos a acurácia (Acc), o índice Kappa, a área sob a curca ROC (AUC) e o F1-Score (F1). A Acc é a aproximação de um resultado com o seu valor de referência real. O Kappa é utilizado para descrever a concordâncias entre 2 ou mais situações [Viera et al. 2005]. A AUC é uma representação gráfica que ilustra a performance de um classificador binário à medida que a variamos o limiar de discriminação. O F1 é uma média ponderada entre a precisão e o recall.

\section{Resultados e Discussão}

Os resultados apresentados nesse trabalho foram obtidos utilizando três algoritmos para obtenção dos mapas de textura, três arquiteturas de CNN e três classificadores. Além disso, de modo a comparar a performance obtida com a combinação dos mapas de textura, executamos o processo utilizando como entrada na CNN os canais de cores do sistema RGB (vermelho, verde e azul). Nas Tabelas 2, 3 e 4 apresentamos os resultados para os classificadores Gaussian Process, Decision Tree e Random Forest, respectivamente.

A Tabela 2 mostra que o melhor resultado foi obtido utilizando a combinação WCG com um Kappa $=0.708 \pm 0.054$. Por outro lado, a menor performance foi obtida pelo canal de cor verde do modelo RGB com Kappa $=0.554 \pm 0.061$.

Os resultados obtidos com o classificadores Decision Tree e Random Forest (Tabelas 3 e 4) tiveram resultado inferior aos obtidos pelo Gaussian Process. Isso mostra que este classificador, mesmo não tendo sido utilizado em nenhum dos trabalhos relacionados levantados, pode ser o algoritmo mais adequado para o problema em questão.

Na Tabela 3 são mostrados os resultados da classificação utilizando o classificador DT, onde podemos observar que o método proposto foi melhor classificado, com relação a utilização das imagem em apenas canais. Já na Tabela 4 as imagens separadas por canais obtiveram uma melhor classificação, porém com diferença miníma da classificação do método proposto. 
Tabela 2. Resultados obtidos pela metodologia proposta utilizando o classificador Gaussian Process.

\begin{tabular}{|c|c|c|c|}
\hline & VGG16 & VGG19 & RESNET-50 \\
\hline & \multicolumn{3}{|c|}{ Vermelho } \\
\hline Acc & $0.813 \pm 0.026$ & $0.829 \pm 0.029$ & $0.828 \pm 0.036$ \\
\hline Kappa & $0.617 \pm 0.049$ & $0.648 \pm 0.061$ & $0.648 \pm 0.071$ \\
\hline AUC & $0.808 \pm 0.023$ & $0.822 \pm 0.033$ & $0.828 \pm 0.032$ \\
\hline \multirow[t]{2}{*}{$\mathrm{F} 1$} & $0.776 \pm 0.029$ & $0.791 \pm 0.038$ & $0.796 \pm 0.040$ \\
\hline & \multicolumn{3}{|c|}{ Verde } \\
\hline Acc & $0.812 \pm 0.034$ & $0.7813 \pm 0.030$ & $0.782 \pm 0.028$ \\
\hline Kappa & $0.606 \pm 0.078$ & $0.553 \pm 0.057$ & $0.554 \pm 0.061$ \\
\hline AUC & $0.801 \pm 0.038$ & $0.775 \pm 0.029$ & $0.777 \pm 0.029$ \\
\hline \multirow[t]{2}{*}{$\mathrm{F} 1$} & $0.762 \pm 0.055$ & $0.743 \pm 0.040$ & $0.746 \pm 0.044$ \\
\hline & \multicolumn{3}{|c|}{ Azul } \\
\hline Acc & 0.7 & $0.757 \pm 0.041$ & $=0.023$ \\
\hline Kappa & $0.482 \pm 0.181$ & $0.498 \pm 0.079$ & 0.042 \\
\hline AUC & $0.741 \pm 0.091$ & $0.747 \pm 0.039$ & 0.020 \\
\hline \multirow[t]{2}{*}{$\mathrm{F} 1$} & $0.647 \pm 0.230$ & $0.700 \pm 0.0478$ & 0.018 \\
\hline & \multicolumn{3}{|c|}{$\mathrm{WCH}$} \\
\hline Acc & \pm 0.035 & $0.725 \pm 0.081$ & 0.030 \\
\hline Kappa & 0.61 & $0.391 \pm 0.256$ & 071 \\
\hline AUC & $0.805 \pm 0.034$ & $0.697 \pm$ & 0.842 \\
\hline \multirow[t]{2}{*}{$\mathrm{F} 1$} & $0.789 \pm 0.033$ & $0.528 \pm 0.343$ & $0.826 \pm 0.031$ \\
\hline & \multicolumn{3}{|c|}{$\mathrm{GCH}$} \\
\hline Acc & 0.74 & $0.812 \pm 0.022$ & \pm 0.020 \\
\hline Kappa & & $0.638 \pm 0.051$ & 0.051 \\
\hline AUC & 0.7 & $0.817 \pm 0.026$ & .021 \\
\hline \multirow[t]{2}{*}{$\mathrm{F} 1$} & 0.73 & $0.83 \pm 0.033$ & $0.869 \pm 0.020$ \\
\hline & \multicolumn{3}{|c|}{ WCG } \\
\hline Acc & $0.803 \pm 0.032$ & $0.748 \pm 0.095$ & $=0.021$ \\
\hline Kappa & 0.5 & $0.415 \pm 0.274$ & \\
\hline AUC & 0.79 & $0.702 \pm$ & $0.856 \pm 0.021$ \\
\hline F1 & $0.762 \pm 0.048$ & $0.536 \pm 0.351$ & $0.829 \pm 0.032$ \\
\hline
\end{tabular}

Quando se muda o vetor de atributos os resultados variam, pois, cada classificador possui parâmetros específicos, estes podem melhorar ou piorar o resultado, dependendo da escolha dos mesmos. Desta forma visando esclarecer esses resultados, para trabalhos futuros pode-se utilizar o grid-search, para estimar os melhores parâmetros de cada classificador.

\section{Conclusão e trabalhos futuros}

Este trabalho apresentou uma metodologia para utilização de mapas de textura para extrair atributos de redes profundas no diagnóstico automático do glaucoma utilizando a base de imagens RIM-ONE-V2. Foram utilizados os algoritmos de textura WAV, GLCM e HOG para geração de mapas de textura de onde foram extraídas características com as CNNs VGG16, VGG19 e RESNET. Ao final as características foram classificadas pelos algoritmos Gaussian Process, Decision Tree e Random Forest.

Os Resultados obtidos foram promissores, em especial para a combinação dos mapas WAV - Imagem em níveis de cinza - GLCM (WCG). Além disso, observamos que a combinação da imagens em níveis de cinza com mapas de textura pode melhorar os resultados quando comparado com o uso somente da imagem. Os resultados indicam que a metodologia, embora necessite de melhorias, pode ser utilizada para auxiliar na detecção precoce do glaucoma. Outro ponto importante que pode ser destacado é que este método 
Tabela 3. Resultados obtidos pela metodologia proposta utilizando o classificador Decision Tree.

\begin{tabular}{c|ccc}
\hline & VGG16 & VGG19 & RESNET-50 \\
\hline & \multicolumn{3}{|c}{ Vermelho } \\
\hline Acc & $0.720 \pm 0.046$ & $0.732 \pm 0.044$ & $0.739 \pm 0.050$ \\
Kappa & $0.427 \pm 0.090$ & $0.458 \pm 0.090$ & $0.465 \pm 0.096$ \\
AUC & $0.716 \pm 0.048$ & $0.728 \pm 0.045$ & $0.731 \pm 0.045$ \\
F1 & $0.665 \pm 0.048$ & $0.700 \pm 0.060$ & $0.688 \pm 0.053$ \\
\hline \multicolumn{3}{|c}{ Verde } \\
\hline Acc & $0.689 \pm 0.027$ & $0.689 \pm 0.039$ & $0.695 \pm 0.036$ \\
Kappa & $0.364 \pm 0.056$ & $0.366 \pm 0.082$ & $0.382 \pm 0.070$ \\
AUC & $0.683 \pm 0.025$ & $0.683 \pm 0.041$ & $0.694 \pm 0.037$ \\
F1 & $0.627 \pm 0.048$ & $0.638 \pm 0.056$ & $0.650 \pm 0.045$ \\
\hline & \multicolumn{3}{|c}{ Azul } \\
\hline Acc & $0.709 \pm 0.049$ & $0.654 \pm 0.033$ & $0.736 \pm 0.039$ \\
Kappa & $0.409 \pm 0.095$ & $0.297 \pm 0.061$ & $0.464 \pm 0.076$ \\
AUC & $0.705 \pm 0.045$ & $0.650 \pm 0.030$ & $0.732 \pm 0.037$ \\
F1 & $0.665 \pm 0.054$ & $0.592 \pm 0.027$ & $0.704 \pm 0.033$ \\
\hline & \multicolumn{3}{|c}{ WCH } \\
\hline Acc & $0.701 \pm 0.042$ & $0.719 \pm 0.047$ & $0.749 \pm 0.022$ \\
Kappa & $0.405 \pm 0.075$ & $0.423 \pm 0.100$ & $0.485 \pm 0.053$ \\
AUC & $0.698 \pm 0.032$ & $0.703 \pm 0.055$ & $0.736 \pm 0.048$ \\
F1 & $0.641 \pm 0.049$ & $0.665 \pm 0.078$ & $0.699 \pm 0.051$ \\
\hline \multicolumn{3}{|c}{ GCH } \\
\hline Acc & $0.673 \pm 0.045$ & $0.698 \pm 0.042$ & $0.689 \pm 0.033$ \\
Kappa & $0.342 \pm 0.095$ & $0.359 \pm 0.091$ & $0.356 \pm 0.061$ \\
AUC & $0.676 \pm 0.044$ & $0.689 \pm 0.047$ & $0.670 \pm 0.020$ \\
F1 & $0.692 \pm 0.052$ & $0.732 \pm 0.042$ & $0.725 \pm 0.039$ \\
\hline & \multicolumn{3}{|c}{ WCG } \\
\hline Acc & $0.689 \pm 0.063$ & $0.682 \pm 0.054$ & $0.729 \pm 0.030$ \\
Kappa & $0.346 \pm 0.124$ & $0.345 \pm 0.122$ & $0.412 \pm 0.088$ \\
AUC & $0.671 \pm 0.065$ & $0.672 \pm 0.069$ & $0.705 \pm 0.040$ \\
F1 & $0.600 \pm 0.093$ & $0.613 \pm 0.089$ & $0.642 \pm 0.63$ \\
\hline & &
\end{tabular}

pode ser extrapolado para outros métodos do estado-da-arte, contribuindo para melhorar os níveis de acurácia desses métodos.

Para trabalhos futuros pretendemos: Implementar outros descritores de textura, tais como o descritor LBP e suas variações; aplicar o método em outras arquiteturas de CNN; combinação da saída de várias CNNs para usar como entrada no algoritmo de classificação; realizar testes em múltiplas bases de imagens; e realizar um treinamento que indique o grau de severidade da doença e não apenas o diagnóstico final.

\section{Agradecimentos}

O método proposto foi apoiado pela Fundação de Amparo à Pesquisa do Piauí (FAPEPI www.fapepi.pi.gov.br).

\section{Referências}

[Ahn et al. 2018] Ahn, J. M., Kim, S., Ahn, K.-S., Cho, S.-H., Lee, K. B., and Kim, U. S. (2018). A deep learning model for the detection of both advanced and early glaucoma using fundus photography. PloS one, 13(11).

[Al-Bander et al. 2017] Al-Bander, B., Al-Nuaimy, W., Al-Taee, M. A., and Zheng, Y. (2017). Automated glaucoma diagnosis using deep learning approach. In 2017 14th 
Tabela 4. Resultados obtidos pela metodologia proposta utilizando o classificador Random Forest.

\begin{tabular}{c|ccc}
\hline & VGG16 & VGG19 & RESNET-50 \\
\hline & \multicolumn{3}{|c}{ Vermelho } \\
\hline Acc & $0.758 \pm 0.055$ & $0.760 \pm 0.060$ & $0.780 \pm 0.031$ \\
Kappa & $0.497 \pm 0.113$ & $0.509 \pm 0.109$ & $0.538 \pm 0.060$ \\
AUC & $0.746 \pm 0.056$ & $0.751 \pm 0.051$ & $0.762 \pm 0.028$ \\
F1 & $0.695 \pm 0.067$ & $0.697 \pm 0.065$ & $0.708 \pm 0.040$ \\
\hline \multicolumn{3}{|c}{ Verde } \\
\hline Acc & $0.720 \pm 0.054$ & $0.745 \pm 0.051$ & $0.734 \pm 0.051$ \\
Kappa & $0.417 \pm 0.106$ & $0.474 \pm 0.099$ & $0.448 \pm 0.091$ \\
AUC & $0.704 \pm 0.054$ & $0.735 \pm 0.047$ & $0.721 \pm 0.042$ \\
F1 & $0.626 \pm 0.078$ & $0.690 \pm 0.055$ & $0.639 \pm 0.062$ \\
\hline & \multicolumn{3}{|c}{ Azul } \\
\hline Acc & $0.702 \pm 0.042$ & $0.712 \pm 0.043$ & $0.775 \pm 0.041$ \\
Kappa & $0.376 \pm 0.078$ & $0.402 \pm 0.081$ & $0.527 \pm 0.090$ \\
AUC & $0.682 \pm 0.037$ & $0.699 \pm 0.038$ & $0.757 \pm 0.048$ \\
F1 & $0.584 \pm 0.068$ & $0.632 \pm 0.050$ & $0.694 \pm 0.071$ \\
\hline & \multicolumn{3}{|c}{ WCH } \\
\hline Acc & $0.709 \pm 0.031$ & $0.687 \pm 0.062$ & $0.742 \pm 0.053$ \\
Kappa & $0.381 \pm 0.081$ & $0.325 \pm 0.136$ & $0.463 \pm 0.100$ \\
AUC & $0.685 \pm 0.042$ & $0.653 \pm 0.063$ & $0.726 \pm 0.043$ \\
F1 & $0.614 \pm 0.053$ & $0.542 \pm 0.100$ & $0.639 \pm 0.077$ \\
\hline & \multicolumn{3}{|c}{ GCH } \\
\hline Acc & $0.719 \pm 0.045$ & $0.668 \pm 0.042$ & $0.712 \pm 0.058$ \\
Kappa & $0.400 \pm 0.096$ & $0.279 \pm 0.099$ & $0.41 \pm 0.100$ \\
AUC & $0.698 \pm 0.042$ & $0.632 \pm 0.048$ & $0.698 \pm 0.046$ \\
F1 & $0.769 \pm 0.052$ & $0.749 \pm 0.049$ & $0.782 \pm 0.049$ \\
\hline \multicolumn{3}{|c}{ WCG } \\
\hline Acc & $0.698 \pm 0.035$ & $0.658 \pm 0.055$ & $0.702 \pm 0.032$ \\
Kappa & $0.352 \pm 0.086$ & $0.289 \pm 0.100$ & $0.394 \pm 0.085$ \\
AUC & $0.678 \pm 0.044$ & $0.635 \pm 0.045$ & $0.698 \pm 0.042$ \\
F1 & $0.602 \pm 0.058$ & $0.519 \pm 0.089$ & $0.589 \pm 0.076$ \\
\hline & \multicolumn{3}{c}{}
\end{tabular}

International Multi-Conference on Systems, Signals \& Devices (SSD), pages 207-210. IEEE.

[Araujo 2018] Araujo, J. (2018). Diagnóstico de glaucoma a partir de imagens de fundo de olho utilizando índices de diversidade. Pós-graduaçao em ciência da computaçao, Universidade Federal do Maranha, Sao Luls.

[Breiman 2001] Breiman, L. (2001). Random forests. Machine learning, 45(1):5-32.

[Breiman et al. 1984] Breiman, L., Friedman, J., Stone, C. J., and Olshen, R. A. (1984). Classification and regression trees. CRC press.

[Carvalho-Júnior et al. 2017] Carvalho-Júnior, A. S. V., de Carvalho Filho, A. O., de Sousa, A., and da Silva Barros, P. (2017). Desenvolvimento de métodos para detecção automática do glaucoma. In Anais do XVII Workshop de Informática Médica. SBC.

[Dalal and Triggs 2005] Dalal, N. and Triggs, B. (2005). Histograms of oriented gradients for human detection.

[Deng et al. 2009] Deng, J., Dong, W., Socher, R., Li, L.-J., Li, K., and Fei-Fei, L. (2009). ImageNet: A Large-Scale Hierarchical Image Database. In CVPR09.

[Haralick et al. 1979] Haralick, R. M. et al. (1979). Statistical and structural approaches to texture. Proceedings of the IEEE, 67(5):786-804. 
[Huang et al. 2016] Huang, G., Liu, Z., and Weinberger, K. Q. (2016). Densely connected convolutional networks. CoRR, abs/1608.06993.

[LENSCOPE 2018] LENSCOPE (2018). Glaucoma: o que é, tipos, sintomas e tratamentos.

[Li et al. 2018] Li, Z., He, Y., Keel, S., Meng, W., Chang, R. T., and He, M. (2018). Efficacy of a deep learning system for detecting glaucomatous optic neuropathy based on color fundus photographs. Ophthalmology, 125(8):1199-1206.

[Mallat 1989] Mallat, S. G. (1989). A theory for multiresolution signal decomposition: the wavelet representation. IEEE Transactions on Pattern Analysis \& Machine Intelligence, (7):674-693.

[Raghavendra et al. 2018] Raghavendra, U., Fujita, H., Bhandary, S. V., Gudigar, A., Tan, J. H., and Acharya, U. R. (2018). Deep convolution neural network for accurate diagnosis of glaucoma using digital fundus images. Information Sciences, 441:41-49.

[Rasmussen 2003] Rasmussen, C. E. (2003). Gaussian processes in machine learning. In Summer School on Machine Learning, pages 63-71. Springer.

[Recommendation ] Recommendation, I. 709-6, parameter values for the hdtv standards for production and international programme exchange. 2015.

[RIMONE 2015] RIMONE, M. I. A. G. (2015). Rim-one r2.

[SILVA et al. 2018] SILVA, C. C. et al. (2018). Diagnóstico de glaucoma em imagens de fundo de olho utilizando estatística espacial.

[SILVA et al. 2017] SILVA, R. R. V., LOPES, J. G. F., ARAÚJO, F. H. D., Medeiros, F. N. S., and USHIZIMA, D. (2017). Visão Computacional em Python Utilizando as Bibliotecas. Scikit-image e Scikit-learn., volume 1 of III Escola Regional de Informática do Piauí. Livro Anais - Artigos e Minicursos., first edition. Sociedade Brasileira de Computação.

[Simonyan and Zisserman 2014] Simonyan, K. and Zisserman, A. (2014). Very deep convolutional networks for large-scale image recognition. arXiv preprint arXiv:1409.1556.

[Stanzione et al. 2019] Stanzione, A., Cuocolo, R., Cocozza, S., Romeo, V., Persico, F., Fusco, F., Longo, N., Brunetti, A., and Imbriaco, M. (2019). Detection of extraprostatic extension of cancer on biparametric mri combining texture analysis and machine learning: preliminary results. Academic radiology.

[Thylefors and Negrel 1994] Thylefors, B. and Negrel, A. (1994). The global impact of glaucoma. Bulletin of the World Health Organization, 72(3):323.

[Viera et al. 2005] Viera, A. J., Garrett, J. M., et al. (2005). Understanding interobserver agreement: the kappa statistic. Fam med, 37(5):360-363. 